\title{
BMJ open Does antiretroviral therapy initiation increase sexual risk taking in Kenyan female sex workers? A retrospective case-control study
}

Elysha Mawji, ${ }^{1}$ Lyle McKinnon, ${ }^{1,2}$ Charles Wachihi, ${ }^{2}$ Duncan Chege, ${ }^{1}$ Paul Thottingal, ${ }^{2,3}$ Anthony Kariri, ${ }^{2}$ Francis Plummer, ${ }^{4,5}$ T Blake Ball, ${ }^{4,5}$ Walter Jaoko, ${ }^{2}$ Elizabeth Ngugi, ${ }^{3}$ Joshua Kimani, ${ }^{2,4}$ Lawrence Gelmon, ${ }^{4}$ Nico Nagelkerke, ${ }^{4,6}$ Rupert Kaul ${ }^{1,2,7}$

To cite: Mawji E, McKinnon L, Wachihi C, et al. Does antiretroviral therapy initiation increase sexual risk taking in Kenyan female sex workers? A retrospective case-control study. BMJ Open 2012;2:e000565. doi:10.1136/

bmjopen-2011-000565

- Prepublication history for this paper is available online. To view this file please visit the journal online (http://dx. doi.org/10.1136/ bmjopen-2011-000565).

EM and LM contributed equally to this work.

Received 4 November 2011 Accepted 6 March 2012

This final article is available for use under the terms of the Creative Commons Attribution Non-Commercial 2.0 Licence; see http://bmjopen.bmj.com

For numbered affiliations see end of article.

Correspondence to Dr Lyle McKinnon; sijuisijali@gmail.com

\section{ABSTRACT}

Objectives: Although antiretroviral therapy (ART) prolongs life and reduces infectiousness, in some contexts, it has been associated with increased sexual risk taking.

Design: Retrospective case-control study.

Setting: Nairobi-based dedicated female sex worker (FSW) clinic.

Participants: HIV-infected FSWs before and after ART initiation ( $n=62)$; HIV-infected and -uninfected control FSWs not starting ART during the same follow-up period $(n=40)$.

Intervention: Initiation of ART.

Primary outcome measures: Self-reported condom use, client numbers and sexually transmitted infection incidence over the study period (before and after ART initiation in cases).

Results: Sexual risk-taking behaviour with casual clients did not increase after ART initiation; condom use increased and sexually transmitted infection incidence decreased in both cases and controls, likely due to successful cohort-wide HIV prevention efforts. Conclusions: ART provision was not associated with increases in unsafe sex in this FSW population.

Female sex workers (FSWs) play a key role in HIV epidemic spread in sub-Saharan Africa ${ }^{1}$ during both early and late epidemic phases ${ }^{2}$ due to multiple partners, high rates of HIV and sexually transmitted infections (STIs), and in many instances, their ability to negotiate safer sex practices are compromised. ${ }^{134}$ HIV prevention in this group may be the highest impact intervention in developing countries. ${ }^{5}$ Therefore, antiretroviral therapy (ART) programmes targeting FSWs may preserve life and reduce HIV transmission at a population level. However, it will be critical to ensure that sexual disinhibition does not counteract these beneficial effects.

\section{ARTICLE SUMMARY}

Article focus

- Impact of starting ART on sexual risk-taking behaviour in FSWs, which could have an important impact on HIV transmission to clients.

Key messages

- ART initiation was not associated with increased risk taking or sexually transmitted infection incidence.

- Both of these declined over time, most likely as a result of risk reduction counselling.

Strengths and limitations of this study

- Strengths include a relevant population, longitudinal follow-up and inclusion of biological measures of risk taking (sexually transmitted infection incidence).

- Limitations include relatively small study groups and limited sampling time points.

ART provision appears to have contradictory effects on HIV transmission. While ART reduces blood and genital tract viral load, and therefore infectiousness, ${ }^{6}$ it has also been associated with reduced safe sex practices $^{7-9}$ and increases in STI incidence in some settings. ${ }^{10}$ Reasons for behavioural disinhibition could be several, including a feeling of improved health after ART initiation. Although this increase has not been the experience to date in resource-poor countries, ${ }^{11}$ it could become an issue as access expands, ${ }^{2}$ and further studies are needed to understand the extent to which these initial observations are generalisable. Mathematical models suggest that if safe sex practices are not maintained, then HIV transmission may increase despite ART-associated reductions in genital tract virus levels. ${ }^{12}$ A recent study in Kenyan sex workers 
with relatively low rates of partner exchange showed no change in risk taking following ART, ${ }^{13}$ but these findings need to be confirmed in a high partner exchange setting.

In the current study, we assessed the impact of ART on sexual behaviour within an established cohort of FSWs from Nairobi, Kenya, in a retrospective case-control study. Institutional Review Boards at Kenyatta National Hospital and the Universities of Manitoba and Toronto approved the study, and all participants gave written informed consent prior to participation. Each participant completed a standardised questionnaire and physical exam every 6 months. Self-reported risk-taking data included the number of clients (casual and regular) and condoms used per week. Percentage condom use with casual clients was calculated from reported client and condom numbers, with maximum usage arbitrarily set at $98 \%$. Risk reduction services provided to all participants included peer- and clinic-based counselling, provision of free condoms and STI management according to Kenyan guidelines. Non-parametric statistical comparisons between groups and within an individual were performed using PASW Statistics 18.0. Self-reported sexual behaviour data were collected at two time points 6 months apart prior to ART initiation and at two time points after: means of continuous variables were calculated for each period, and changes within an individual compared by using the Wilcoxon signed-rank test. Categorical variables were compared by Mann-Whitney test.

\section{RESULTS}

All HIV-infected FSWs initiating ART during 2001-2006 with $\geq 1$ year of follow-up were included as cases $(n=62)$. Since sexual risk taking may change over time in the cohort as a whole, HIV-infected ART naive and HIVuninfected FSWs enrolled in the cohort for a similar duration were selected as controls $(n=20$ each). Cases and controls were generally comparable at baseline, but cases reported higher condom use with casual clients (mean $97.8 \%$ vs $95.7 \%, \mathrm{p}=0.01$ ) and had a trend to a lower number of unprotected sex acts over the past year (23.0 vs $34.1, p=0.14$, table 1 ). The proportion of participants reporting a regular client was similar (cases
$58 \%$ vs controls $65 \%, \mathrm{p}=0.62)$, and condoms were rarely used with regular clients (cases $23.2 \%$ vs controls $9.6 \%$, $\mathrm{p}=0.13)$.

No significant change was seen in casual client numbers during the year after starting ART in cases (19.9/week pre-ART vs 21.9 post-ART, $\mathrm{p}=0.17$, table 2 ), in condom use with casual clients $(97.8 \%$ both pre-ART and post-ART, $\mathrm{p}=0.66$ ) or in the number of unprotected sex acts with casual clients (23.7/year pre-ART vs 24.7 post-ART, $\mathrm{p}=0.26$ ). Condom use increased in controls (mean $95.7 \%$ vs $97.6 \%, \mathrm{p}=0.14$ ), so that although casual client numbers increased over the study period from 19.4 to 24.5 per week $(p=0.002)$, there was a trend to reduced unprotected sex acts with casual clients during the latter year (34.1 vs 29.5, $\mathrm{p}=0.10$ ).

There was some evidence to suggest an increase in condom use with regular clients in both cases (3.91 vs $3.98, \mathrm{p}=0.06)$ and controls (3.80 vs $3.93, \mathrm{p}=0.05)$. The mean number of regular clients remained unchanged in cases $(0.64$ vs $0.43, \mathrm{p}=0.13)$ and controls $(0.55$ vs 0.60 , $\mathrm{p}=0.25)$, although controls had more regular partners during follow-up ( 0.60 vs $0.43, p=0.04)$. It should be noted that condom use with regular partners remained infrequent in all groups; specific interventions to increase this in FSW populations could have important public health benefits.

Social desirability or fear of being taken off ART could associate with false reporting of sexual behaviour by FSWs, and so STI rates were also examined. Combining Trichomonas vaginalis and Neisseria gonorrhoea data in all participants, 12/102 FSWs were STI positive during the year pre-ART compared with $8 / 102$ post-ART $(\mathrm{p}=0.346)$. This included $6 / 62$ cases and $6 / 40$ controls pre-ART and $4 / 62$ cases and 4/40 controls post-ART. The period prevalence of $N$ gonorrhoea declined from $4.6 \%$ to $1.1 \%$ in the periods before and after ART initiation $(\mathrm{p}=0.02$, Wilcoxon test); this decrease was apparent in both cases $(6 / 78,7.7 \%$, pre-ART vs $1 / 76,1.3 \%$, post-ART) and controls $(3 / 116,2.6 \%$, pre-ART vs $1 / 110,0.9 \%$, post-ART).

In summary, we found no increase in sexual risk taking in FSWs who initiated ART, using either self-reported behaviour or STI rates. This was in the context of clinicand peer-based risk reduction services offered to all

Table 1 Baseline characteristics of study groups

\begin{tabular}{lccc}
\hline Characteristic (median, range) & $\begin{array}{l}\text { Cases starting } \\
\text { ART (N=62) }\end{array}$ & $\begin{array}{l}\text { HIV-infected } \\
\text { controls (N=20) }\end{array}$ & $\begin{array}{l}\text { HIV-uninfected } \\
\text { controls (N=20) }\end{array}$ \\
\hline Age (years) & $41(26-61)$ & $38(27-48)$ & $41(27-61)$ \\
Duration of sex work (years) & $14(2-41)$ & $13(1-28)$ & $11(2-33)$ \\
Clients per week & $16(4-80)$ & $20(3-51)$ & $19(2-45)$ \\
Condom use (\%) & $98(86-98)^{*}$ & $98(75-98)$ & $98(50-98)$ \\
Unprotected sex acts (past year) & $16(4-160)$ & $21(3-195)$ & $24(4-171)$ \\
Regular partner (proportion) & $36 / 62(58 \%)$ & $12 / 20(60 \%)$ & $14 / 20(70 \%)$ \\
Condom with regular partner (\%) & 23 & 8 & 11 \\
CD4 T cell count & $205(70-1028)^{*}$ & $557(243-1082)$ & $1100(631-1692)$ \\
\hline${ }^{*}$ Mann-Whitney p $\leq 0.01$ for cases versus controls. & &
\end{tabular}


Table 2 Longitudinal assessment of risk taking and sexually transmitted infection prevalence in female sex workers over time

\begin{tabular}{lcc}
\hline Variable & Before ART & After ART \\
\hline Cases & & 24.72 \\
No. unprotected acts with casual partners/year & 23.05 & 3.98 \\
Condom use with casual clients (scale 1-4) & 3.91 & 0.39 \\
Condom use with regular clients (scale 1-4) & 0.24 & 4.12 \\
Mean number of casual clients per day & 3.93 & 0.43 \\
Mean number of regular clients per day & 0.64 & $6.5 \%$ \\
NG/TV period prevalence (\%) & $9.7 \%$ & $1.3 \%$ \\
NG period prevalence (\%) & $7.7 \%$ & 0.06 \\
Controls & 34.08 & 29.49 \\
No. unprotected acts with casual partners/year & 3.80 & 3.93 \\
Condom use with casual clients (scale 1-4) & 0.12 & 0.13 \\
Condom use with regular clients (scale 1-4) & 4.11 & 4.43 \\
Mean number of casual clients per day & 0.55 & 0.60 \\
Mean number of regular clients per day & $15 \%$ & $10 \%$ \\
NG/TV period prevalence (\%) & $2.6 \%$ & 0.13 \\
NG period prevalence (\%) & $0.9 \%$ \\
\hline ART, antiretroviral therapy; NG, Neisseria gonorrhoea; TV, Trichomonas vaginalis. & 0.03 \\
\end{tabular}

participants. The latter may be important since studies in Uganda $^{14}$ and Kenya ${ }^{13}{ }^{15}$ showed increases in safe sex practices following ART initiation in conjunction with risk reduction services, while in Cote d'Ivoire, there was an increase in unprotected sex post-ART in their absence. ${ }^{16}$ This suggests that ART should be combined with risk reduction services where possible. A prior Kenyan FSW study also reported no increased risk after ART initiation, ${ }^{13} 15$ although in that cohort less than a third of participants reported more than one partner over the past week compared with a mean of $>20$ casual clients in our study.

Several factors may potentially impact sexual practices in FSW participants, including nationwide HIV education campaigns that could be associated with national declines in HIV prevalence ${ }^{17} 18$ and a cohort-wide risk reduction programme. ${ }^{19}$ Although we found no change in HIV risk behaviour after starting ART, these factors could have masked increases in risk relative to other cohort participants after ART initiation. To rule out this possibility, we assessed a control group of 40 FSWs followed over a similar time span, both HIV-infected ART naive and HIV-uninfected FSWs, and found no evidence that this was the case.

In conclusion, we found no evidence for alterations in sexual behaviour after starting ART in Kenyan FSWs with very high partner exchange rates. FSWs through their profession may act as an important core HIV transmission group in the region. Since ART has been shown to reduce HIV transmission, ${ }^{20} 21$ ART provision for FSWs in conjunction with risk reduction services should be considered an important strategy to reduce HIV transmission at a population level.

\section{Author affiliations}

${ }^{1}$ Department of Medicine, University of Toronto, Toronto, Ontario, Canada

${ }^{2}$ Department of Medical Microbiology, University of Nairobi, Nairobi, Kenya

${ }^{3}$ Department of Community Health, University of Nairobi, Nairobi, Kenya
${ }^{4}$ Department of Medical Microbiology, University of Manitoba, Winnipeg, Manitoba, Canada

${ }^{5}$ National Microbiology Lab, Public Health Agency of Canada, Winnipeg, Manitoba, Canada

${ }^{6}$ Department of Community Medicine, United Arab Emirates University, Al Ain, United Arab Emirates

${ }^{7}$ Department of Medicine, University Health Network, Toronto, Ontario, Canada

Acknowledgements We would like to thank the staff at Majengo clinic and the Kenyan AIDS Control Project for supporting the study and the patients for their willingness to participate.

Contributors EM, PT, WJ, JK, LG and RK designed the study. EM, LM, AK, NN and RK analysed the data. CW and JK managed the clinical cohorts. All authors contributed to the writing and editing of the manuscript.

Funding This research was supported by grants from the Canadian Institutes of Health Research (RK; HET-85518 and MOP-89983); patient care activities and the purchase of antiretroviral drugs were funded by the President's Emergency Plan for AIDS Relief (PEPFAR). Salary support was provided by the Canadian Institutes of Health Research and the International Infectious

Diseases and Global Health Training Program (LM) and the Canada Research Chair Program (RK).

\section{Competing interests None.}

\section{Patient consent Obtained.}

Ethics approval Ethics approval was provided by the University of Manitoba, University of Toronto, Kenyatta National Hospital.

Provenance and peer review Not commissioned; externally peer reviewed.

Data sharing statement There are no additional data available.

\section{REFERENCES}

1. Plummer FA, Nagelkerke NJ, Moses S, et al. The importance of core groups in the epidemiology and control of HIV-1 infection. AIDS 1991;5(Suppl 1):S169-76.

2. Chen $L$, Jha $P$, Stirling $B$, et al. Sexual risk factors for HIV infection in early and advanced HIV epidemics in sub-Saharan Africa: systematic overview of 68 epidemiological studies. PLoS One 2007;2:e1001.

3. Kreiss JK, Koech D, Plummer FA, et al. AIDS virus infection in Nairobi prostitutes. Spread of the epidemic to East Africa. N Engl J Med 1986;314:414-18.

4. Lowndes $\mathrm{CM}$, Alary $\mathrm{M}$, Meda $\mathrm{H}$, et al. Role of core and bridging groups in the transmission dynamics of HIV and STIs in Cotonou, Benin, West Africa. Sex Transm Infect 2002;78(Suppl 1):i69-77.

5. Jha P, Nagelkerke JD, Ngugi EN, et al. Public health. Reducing HIV transmission in developing countries. Science 2001;292:224-5. 
6. Montaner JS, Hogg R, Wood E, et al. The case for expanding access to highly active antiretroviral therapy to curb the growth of the HIV epidemic. Lancet 2006;368:531-6.

7. Desquilbet L, Deveau C, Goujard C, et al. Increase in at-risk sexual behaviour among HIV-1-infected patients followed in the French PRIMO cohort. AIDS 2002;16:2329-33.

8. Ostrow DE, Fox KJ, Chmiel JS, et al. Attitudes towards highly active antiretroviral therapy are associated with sexual risk taking among HIV-infected and uninfected homosexual men. AIDS 2002;16:775-80.

9. Bezemer D, de Wolf F, Boerlijst MC, et al. A resurgent HIV-1 epidemic among men who have sex with men in the era of potent antiretroviral therapy. AIDS 2008;22:1071-7.

10. Scheer S, Chu PL, Klausner JD, et al. Effect of highly active antiretroviral therapy on diagnoses of sexually transmitted diseases in people with AIDS. Lancet 2001;357:432-5.

11. Kennedy C, O'Reilly K, Medley A, et al. The impact of HIV treatment on risk behaviour in developing countries: a systematic review. AIDS Care 2007;19:707-20.

12. Wilson DP, Law MG, Grulich AE, et al. Relation between HIV viral load and infectiousness: a model-based analysis. Lancet 2008;372:314-20.

13. McClelland RS, Graham SM, Richardson BA, et al. Treatment with antiretroviral therapy is not associated with increased sexual risk behavior in Kenyan female sex workers. AIDS 2010;24:891-7.
14. Bunnell R, Ekwaru JP, Solberg P, et al. Changes in sexual behavior and risk of HIV transmission after antiretroviral therapy and prevention interventions in rural Uganda. AIDS 2006;20:85-92.

15. Luchters S, Sarna A, Geibel S, et al. Safer sexual behaviors after 12 months of antiretroviral treatment in Mombasa, Kenya: a prospective cohort. AIDS Patient Care STDS 2008;22:587-94.

16. Diabate $\mathrm{S}$, Alary M, Koffi CK. Short-term increase in unsafe sexual behaviour after initiation of HAART in Cote d'Ivoire. AIDS 2008:22:154-6.

17. National AIDS Control Council OotP. UNGASS 2008 country report for Kenya. In: National AIDS Control Council N, ed. 2008.

18. Kimani J, Kaul R, Nagelkerke NJ, et al. Reduced rates of HIV acquisition during unprotected sex by Kenyan female sex workers predating population declines in HIV prevalence. AIDS 2008:22:131-7.

19. Kaul R, Kimani J, Nagelkerke NJ, et al. Reduced HIV risk-taking and low HIV incidence after enrollment and risk-reduction counseling in a sexually transmitted disease prevention trial in Nairobi, Kenya. $J$ Acquir Immune Defic Syndr 2002;30:69-72.

20. Attia S, Egger M, Muller M, et al. Sexual transmission of HIV according to viral load and antiretroviral therapy: systematic review and meta-analysis. AIDS 2009;23:1397-404.

21. Donnell D, Baeten JM, Kiarie J, et al. Heterosexual HIV-1 transmission after initiation of antiretroviral therapy: a prospective cohort analysis. Lancet 2010;375:2092-8. 